\section{Failure of adipocyte differentiation causes type II diabetes mellitus?}

\begin{abstract}
$A$ nand and $\mathrm{Chada}^{1}$ have reported that A knockouts of Hmgic prevent adipocyte differentiation and therefore might serve as a target for obesity. Prevention of adipocyte differentiation, however, is destined to exchange obesity for diabetes and suggests an interesting hypothesis: that type II diabetes is the result of the inability of the adipose organ to expand to accommodate excess calories and that type II diabetes in the centrally obese, in spite of their unlikely phenotype, is a form of lipodystrophy. If the adipose organ is unable to accommodate excess energy, the calories are stored in the liver, muscles and, with the development of diabetes, in the blood and urine. This is most clearly seen in acquired total lipodystrophy where a positive energy balance begets diabetes ${ }^{2}$, and is revealed in obese type II diabetics when, early in their disease, caloric restriction reverses the diabetes.

This is supported by Gavrilova et al. ${ }^{3}$, who reversed the diabetes in a mouse model of lipodystrophy by surgically implanting adipose tissue. It is supported by Okuno et al. ${ }^{4}$, who found that thiazolidinediones improved insulin sensitivity
\end{abstract}

in Zucker rats by increasing the number of new adipocytes. It is supported in studies in obese Pima Indians, in which the best correlation with the onset of diabetes is with adipocyte size, suggesting difficulty in differentiating new ones ${ }^{5}$. It is supported by the observation that insulin sensitivity during overfeeding correlates with the recruitment of new adipocytes ${ }^{6}$, and that the in vitro yield of newly differentiated adipocytes is greater in lean than in obese subjects.

Adipocytes are terminal cells that when filled to capacity $(\sim 3 \mu \mathrm{g}$ lipid/cell) are extremely insulin resistant. Therefore, excess energy intake must be balanced by the expansion of the adipose organ. If not, the energy is stored in the liver, muscle and blood and is accompanied by insulin resistance as reported by Randle and colleagues ${ }^{7}$. Hepatic steatosis and excess lipid in muscle and pancreas is characteristic of obese diabetics ${ }^{8}$. These abnormal deposits of lipid may lead to the trilogy of defects characteristic of type II diabetes mellitus: over-production of glucose by the liver, muscle insulin resistance and blunted secretion of insulin by the pancreas. Sev- eral laboratories now support this, including those of McGarry ${ }^{8}$ and Unger ${ }^{9}$. Therefore, any scenario that targets the destruction of adipocytes or prevents their differentiation in the face of excess caloric intake is doomed to exchange obesity for type II diabetes mellitus.

Thirty years ago, Knittle and Hirsch ${ }^{10}$ suggested that too many adipocytes predisposed to obesity. I suggest that too few adipocytes predisposes to type II diabetes mellitus. This could explain the lower prevalence of type II diabetes in the generalized and more hypercellular obese than in the centrally obese, who for genetic or environmental reasons have lost the ability to accommodate excess energy by differentiating new adipocytes.

\section{Elliot Danforth Jr}

University of Vermont, Underhill, Vermont, USA (e-mail: edanforth@att.net).

1. Anand, A. \& Chada, K. Nature Genet. 24, 377-380 (2000)

2. Robbins, D.C. et al. Metabolism 28, 908-916 (1979).

3. Gavrilova, O. et al. J. Clin. Invest. 105, 271-278 (2000).

4. Okuno, A. et al. J. Clin. Invest. 101, 1354-1361 (1998).

5. Paolisso, G. et al. Diabetologia 38, 1213-1217 (1995).

6. Kashiwagi, A. et al. Metabolism 34, 364-370 (1985)

7. Randle, P.J., Kales, C.N., Garland, P.B. \& Newshome, E.A. Lancet 1, 785-789 (1963).

8. McGarry, J.D. \& Dobbins, R.L. Diabetologia 42 128-138 (1999)

9. Unger, R.H., Shou, Y. \& Orci, L. Proc. Natl Acad. Sci. USA 96, 2327-2332 (1999)

10. Knittle, J.L. \& Hirsch, J. J. Clin. Invest. 47, 2091-2097 (1968).

\section{Sampling SNPs}

Several major single-nucleotide $\checkmark$ polymorphism (SNP) discovery projects have been launched recently ${ }^{1}$. The SNP Consortium alone has released 102,719 SNPs (http://snp.cshl.org). More recently, 664,846 SNPs based on the clone overlaps ${ }^{2}$

$a$

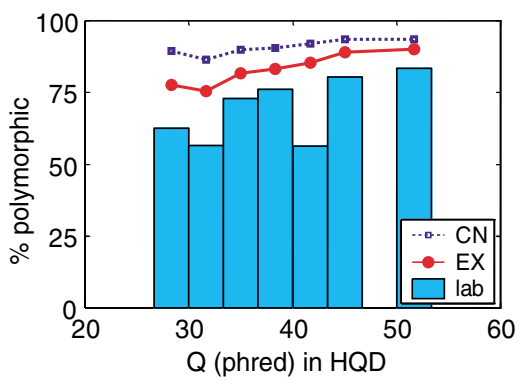

from the Human Genome Project were submitted by groups at the Sanger Center and at Washington University. So far, few of these SNPs have been validated. Moreover, validation rates have been derived by resequencing the same DNA samples used for

\section{$b$}

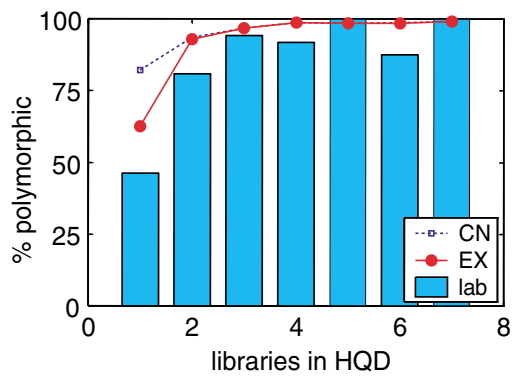

Fig. 1 Polymorphism rate in second sample, plotted against Phred quality (a) and number of libraries in HQD (b). The dotted and solid lines show the predicted rates for the constant (CN) and expanded (EX) population models, respectively, whereas the histogram bars show the observed rates. the initial discovery. This approach controls for experimental artefacts in the discovery process, but does not control for the presence of segregating sites with rare alleles. Because such sites comprise most of the sites that vary in the human population, they may represent a significant fraction of all sites detected as 'SNPs'. The rare alleles are unlikely to be observed again in a second sample, and therefore these sites will not be useful for genetic studies.

To investigate this phenomenon, we surveyed the expressed sequence tag (EST) databases $^{3}$ for high-quality discrepancies (HQDs), designed PCR primers to amplify these putative SNPs from genomic DNA and re-sequenced them in a second DNA sample ${ }^{4}$. We applied stringent data quality restrictions on the HQDs to minimize the number of sequencing artefacts. Almost all selected HQDs had two or more high-quality EST reads for both alleles, with an overall Phred quality ${ }^{5,6}$ of greater than or equal to Q30 (that is, an expected error rate $\left.\leq 10^{-3}\right)$. We also inspected the EST traces visually. After PCR testing, 436 putative SNPs were available. These were re- 